\title{
Dual array EEG-fMRI : An approach for motion artifact suppression in EEG recorded simultaneously with fMRI
}

\section{Klovatch-Podlipsky, Ilana}

2016-11-15

Klovatch-Podlipsky , I, Gazit , T, Fahoum , F , Tsirelson, B , Kipervasser , S , Kremer , U , Ben-Zeev , B , Goldberg-Stern , H , Eisenstein , O , Harpaz , Y, Levy , O , Kirschner , A , Neufeld , M Y , Fried , I , Hendler , T \& Medvedovsky , M 2016 , ' Dual array EEG-fMRI : An approach for motion artifact suppression in EEG recorded simultaneously with fMRI ' , Neurolmage , vol. 142 , pp. 674-686 . https://doi.org/10.1016/j.neuroimage.2016.07.014

http://hdl.handle.net/10138/229300

https://doi.org/10.1016/j.neuroimage.2016.07.014

publishedVersion

Downloaded from Helda, University of Helsinki institutional repository.

This is an electronic reprint of the original article.

This reprint may differ from the original in pagination and typographic detail.

Please cite the original version. 
Technical Note

\title{
Dual array EEG-fMRI: An approach for motion artifact suppression in EEG recorded simultaneously with fMRI
}

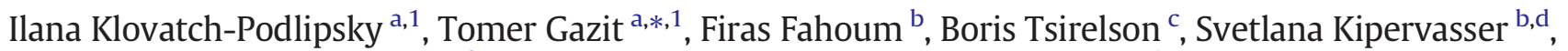 \\ Uri Kremer ${ }^{\mathrm{e}}$, Bruria Ben-Zeev ${ }^{\mathrm{f}}$, Hadassah Goldberg-Stern ${ }^{\mathrm{g}}$, Orna Eisenstein ${ }^{\mathrm{h}}$, Yuval Harpaz ${ }^{\mathrm{i}}$, Ory Levy ${ }^{\mathrm{a}}$, \\ Adi Kirschner ${ }^{\mathrm{a}}$, Miriam Y. Neufeld ${ }^{\mathrm{b}, \mathrm{d}}$, Itzhak Fried ${ }^{\mathrm{j}, \mathrm{k}}$, Talma Hendler ${ }^{\mathrm{a}, \mathrm{d}, \mathrm{m}, \mathrm{n}}$, Mordekhay Medvedovsky ${ }^{\mathrm{a}, \mathrm{l}}$ \\ a Tel-Aviv Center for Brain Functions, Whol Institute for Advance Imaging, Tel Aviv Sourasky Medical Center, Israel \\ b EEG and Epilepsy Unit, Department of Neurology, Tel Aviv Sourasky Medical Center, Israel \\ c School of Mathematics, Tel Aviv University, Israel \\ d Sackler School of Medicine, Tel Aviv University, Israel \\ e Pediatric Epilepsy Unit, Tel Aviv Sourasky Medical Center, Tel Aviv University, Tel Aviv, Israel \\ ${ }^{f}$ Pediatric Neurology Unit, Edmond and Lilly Safra Children's Hospital, Sheba Medical Center, Ramat-Gan, Israel \\ 'Schneider Children's Medical Center of Israel, Petach Tikvah, Israel \\ h Department of Radiology, Tel Aviv Sourasky Medical Center, Israel \\ ${ }^{i}$ Gonda Multidisciplinary Brain Research Center, Bar-Ilan University, Ramat-Gan, Israel \\ ${ }^{\mathrm{j}}$ Department of Neurosurgery, David Geffen School of Medicine and Semel Institute for Neuroscience and Human Behavior, University of California, Los Angeles, CA, USA \\ ${ }^{\mathrm{k}}$ Functional Neurosurgery Unit, Tel-Aviv Medical Center, Israel \\ ${ }^{1}$ Helsinki University Central Hospital, Helsinki, Finland \\ ${ }^{\mathrm{m}}$ School of Psychological Sciences, Tel-Aviv University, Israel \\ ${ }^{n}$ Sagol School of Neuroscience, Tel-Aviv University, Israel
}

\section{A R T I C L E I N F O}

\section{Article history:}

Received 17 December 2015

Accepted 6 July 2016

Available online 9 July 2016

\section{Keywords:}

EEG

fMRI

ICA

Ballistocardiogram

Artifact

\section{A B S T R A C T}

Objective: Although simultaneous recording of EEG and MRI has gained increasing popularity in recent years, the extent of its clinical use remains limited by various technical challenges. Motion interference is one of the major challenges in EEG-fMRI. Here we present an approach which reduces its impact with the aid of an MR compatible dual-array EEG (daEEG) in which the EEG itself is used both as a brain signal recorder and a motion sensor. Methods: We implemented two arrays of EEG electrodes organized into two sets of nearly orthogonally intersecting wire bundles. The EEG was recorded using referential amplifiers inside a 3 T MR-scanner. Virtual bipolar measurements were taken both along bundles (creating a small wire loop and therefore minimizing artifact) and across bundles (creating a large wire loop and therefore maximizing artifact). Independent component analysis (ICA) was applied. The resulting ICA components were classified into brain signal and noise using three criteria: 1) degree of two-dimensional spatial correlation between ICA coefficients along bundles and across bundles; 2) amplitude along bundles vs. across bundles; 3 ) correlation with ECG. The components which passed the criteria set were transformed back to the channel space. Motion artifact suppression and the ability to detect interictal epileptic spikes following daEEG and Optimal Basis Set (OBS) procedures were compared in 10 patients with epilepsy.

Results: The SNR achieved by daEEG was $11.05 \pm 3.10$ and by OBS was $8.25 \pm 1.01(p<0.00001)$. In 9 of 10 patients, more spikes were detected after daEEG than after OBS $(p<0.05)$.

Significance: daEEG improves signal quality in EEG-fMRI recordings, expanding its clinical and research potential.

(c) 2016 Elsevier Inc. All rights reserved.
Abbreviations: (daEEG), dual array EEG; (OBS), Optimal Basis Set; (ICA), independent component analysis; (BCG), ballistocardiogram; (BOLD), blood oxygenation level dependant.

* Corresponding author.

E-mail address: Tomergazit@gmail.com (T. Gazit).

1 Authors contributed equally to the study.

\section{Introduction}

Simultaneous Electroencephalography and functional magnetic resonance imaging (EEG-fMRI) is a neuroimaging technique that is being increasingly used in both research and clinical settings. The high temporal resolution of EEG complements the high spatial resolution of fMRI. However, combining the two modalities simultaneously imposes significant interference on the EEG signal due to the changing magnetic field 
of the MRI (gradient interference) and due to movements inside the magnetic field (motion interference). While gradient interference has a predictable time course, and can therefore be successfully suppressed (Allen et al., 2000), motion interference is usually harder to suppress. When subjects do not actively move their head or face, motion interference largely results from the ballistocardiogram (BCG), which comprises at least three different components: cardiac-pulse-induced head rotation, pulsatile scalp expansion and Hall voltages (Mullinger et al., 2013). Although the BCG is less predictable compared to gradient interference, it can usually be mostly suppressed due to its repetitive nature (Allen et al., 1998). However, other motion interference types, which are not related to BCG (non-BCG), may also exist in the data and their suppression is more challenging. Non-BCG motion interference results from vibrations generated by, or transmitted to the MRI scanner, and/ or from small movements of the subject (such as fine tremor and even breathing). The non-BCG interference is usually less prominent than BCG interference. Nevertheless, it can disturb the EEG interpretation, especially when the EEG waveforms (e.g. epileptic spikes) have relatively low amplitude and the EEG is recorded inside an MR-scanner with a relatively high magnetic field (e.g. $3 \mathrm{~T}$ ). Epileptic waveform detection is not always trivial even in standard (out-of-scanner) EEG recordings, and can be especially challenging in EEG-fMRI. In some circumstances the difficulty in obtaining reasonable signal-to-noise ratio can be an obstacle to EEG-fMRI use in pre-surgical epilepsy workup. The MR scanner vibrations can be suppressed to some degree but not completely eliminated; and the degree of vibration control varies between different scanner types and at different installation sites.

The issue of non-BCG motion artifacts was addressed by several studies to date. Bonmassar et al. (2002) used a piezoelectric-transducer fixed to the area of the temporal artery as a motion sensor for BCG and nonBCG motions. Masterton et al. (2007) demonstrated suppression of both BCG and non-BCG interference by implementing three wire loops fixed to the EEG cap and connected to the bipolar amplifier as a motion sensor independent from EEG. Luo et al. (2014) and Chowdhury et al. (2014) reported an artifact suppression approach which is based on the reference layer of electrodes embedded into the conductive material. These techniques however require additional MR-compatible equipment. Jorge et al. (2015) reported a method in which some of the EEG electrodes were kept isolated from the scalp and connected by wires with resistors to the reference electrode, resulting in the creation of wire loops which served as motion sensors. This approach did not require additional equipment, rather only minimal changes of commercially available MR-compatible EEG array. The present work introduces the dual array EEG (daEEG) approach which employs the EEG array itself as a motion sensor. This approach is based on the fact that motion artifacts are influenced by the size of the loop between EEG wires (connecting the electrodes to the amplifier) while brain signals are not. Thus we constructed an EEG net, in which signals are measured by electrodes arranged in both small and large loops, allowing a separation between signal and motion artifact. Our hypothesis is that using this new EEG arrangement, along with its accompanying algorithm to separate signal and noise, will provide a valuable method for improved suppression of motion induced artifacts when measured inside the MRI.

The daEEG approach employs a comparison of brain signals measured by adjacent electrodes over the whole head. Mild variations of signals between adjacent electrodes are possible. However, due to skull smearing effect, these variations are small and in most cases do not substantially influence the spatial correlation, particularly when taking into account that the correlation is calculated over the whole head rather than between individual electrodes.

\section{Methods}

The study was approved by the Tel-Aviv Sourasky Medical Center (TASMC) Ethical Review Board. Written informed consent for participation in the study was obtained from all patients or their guardians.

\section{Patients}

This study is based on the daEEG-fMRI recordings and epileptic spike analysis of pharmacoresistant patients with epilepsy. The patients considered for this study (a) suffered from pharmacoresistant focal epilepsy (b) were being considered for neurosurgery and (c) had no known risk factors for participating in an fMRI study.

Between August 2012 and October 2013, 24 sequential patients with epilepsy underwent daEEG-fMRI recording. Fourteen patients were excluded from this study: In three cases the recording failed due to technical reasons. In six patients no epileptic signals were found. In three patients epileptic rhythms but not spikes were observed. Two patients had less than ten epileptic spikes (insufficient for accurate statistical evaluation). The remaining 10 patients were included in the study (Table 1).

\section{Description of the daEEG concept}

EEG array as a motion sensor: EEG acquisition was performed in a referential montage and subsequently recalculated into a virtual bipolar montage.

As a first step, the EEG electrodes were grouped along longitudinal and transverse lines (as can be seen in Fig. 1A). The wires of the electrodes along a single line were bundled together to reduce the size of the loop created.

First consider an array of several parallel transverse bundles of EEG cables (array I - transverse = lines in Fig. 1 comprising both dark blue and dark magenta bundles). Taking bipolar measurements along such bundles (such as in E1-E2 bipolar measurement in Fig. 1b) minimize the area within the loop created by the electrode cables; therefore motion interference is also minimized (Fig. 1b). In contrast when the bipolar measurements are taken across different bundles (such as in the in E1-E5 bipolar measurement in Fig. 1 or in Fig. 1c and d) the area within the loop created by electrode cables is larger, creating larger motion interference. Thus, using array I of only transverse bundles, we have created bipolar measurements with both small and large motion interference. However, since the orientation of the across-bundles bipolar measurement in this array is longitudinal (as in E1-E5 bipolar measurement), whereas the orientation of the along-bundles bipolar measurement (such as in E1-E2 bipolar measurement) is transverse, they cannot be compared for the purpose of differentiating signal to noise. This is due to the fact that differences between along and across measurements can be caused by increased motion artifact in the across-bundle measurement but also because of differences in brain signal gradient orientation (for example a brain signal gradient with longitudinal orientation will not be observed in transverse bipolar measurement).

Therefore, consider an additional array of several parallel longitudinal bundles of EEG cables (array II, longitudinal $\|$ lines in Fig. 1 comprising of both light cyan and light magenta bundles) whose EEG sampling lines are oriented orthogonally to the sampling lines of array I bundles. From these two arrays we can create four sets of bipolar measurements:

1. Along bundles of array I (as in E1-E2, transverse).

2. Along bundles of array II (as in E3-E6, longitudinal).

3. Across bundles of array I (as in E1-E5, longitudinal).

4. Across bundles of array II (as in E3-E4, transverse).

Sets 1 and 2 are the measurements with minimized interference, in orthogonal directions, and sets 3 and 4 - with maximized interference, also in orthogonal directions. Bipolar measurement of the first set (Fig. 1b) can be compared with those of the fourth set (Fig. 1c or d) since they are in the same orientation but differentiate in the size of the wire loop. Similarly, bipolar measurement of the second set can be compared with those of the third set. Since the data components representing brain activity are not influenced by cable orientation, they are expected to yield similar measurements along and across 
Table 1

Patient characteristics.

\begin{tabular}{|c|c|c|c|c|c|c|c|c|c|c|c|c|}
\hline $\begin{array}{l}\text { Patient } \\
\#\end{array}$ & Age & Gender & Onset & $\begin{array}{l}\text { Epilepsy localization (lesion in } \\
\text { MRI) }\end{array}$ & MED & $\begin{array}{l}\text { Ictal } \\
\text { EEG }\end{array}$ & $\begin{array}{l}\text { Interictal } \\
\text { EEG }\end{array}$ & Neurophsycho-logy & $\begin{array}{l}\text { Interictal } \\
\text { PET-FDG }\end{array}$ & MEG & $\begin{array}{l}\text { iEEG ictal } \\
\text { onset }\end{array}$ & Pathology \\
\hline 1 & 27 & $\mathrm{~F}$ & 17 & R F (atrophy) & CBZ, LEV & $\mathrm{RF}$ & $\begin{array}{l}\mathrm{BiF}(\mathrm{R}> \\
\mathrm{L})\end{array}$ & $\mathrm{F}$ & Bi T HM & R F & $\mathrm{N} / \mathrm{A}$ & $\mathrm{N} / \mathrm{A}$ \\
\hline 2 & 28 & $\mathrm{~F}$ & 18 & $\mathrm{R}$ TP (NL) & TPX & $\mathrm{R}$ pT & R T & Bi FT & R T HM & $\begin{array}{l}\text { R } \\
\text { FTI }\end{array}$ & $\mathrm{N} / \mathrm{A}$ & $\mathrm{N} / \mathrm{A}$ \\
\hline 3 & 26 & M & 12 & $\mathrm{R} F C P(\mathrm{CB})$ & $\mathrm{CBZ}$ & R FC & R FC & Normal & $\mathrm{Bi} \mathrm{T}(\mathrm{L}>\mathrm{R}) \mathrm{HM}$ & R FC & $\mathrm{N} / \mathrm{A}$ & $\mathrm{N} / \mathrm{A}$ \\
\hline 4 & 40 & M & 31 & $\mathrm{RT}(\mathrm{CB})$ & LTG, OXC & $\mathrm{R}$ aT & R T & Bi FT & $\mathrm{Bi} \mathrm{T}(\mathrm{L}>\mathrm{R}) \mathrm{HM}$ & $\mathrm{R} \mathrm{T}$ & $\operatorname{BiT}(\mathrm{R}>\mathrm{L})$ & $\mathrm{N} / \mathrm{A}$ \\
\hline 5 & 13 & M & 4 & R FCP (FCD) & LCS, LEV & R CP & R FCP & Normal & Normal & $\begin{array}{l}\mathrm{Bi} \\
\mathrm{FC}\end{array}$ & $\mathrm{N} / \mathrm{A}$ & FCD2b \\
\hline 6 & 16 & $\mathrm{~F}$ & 12 & $\mathrm{R}$ TP (NL) & CBZ, CLB & R TP & R TP & Normal & R P HM & R P & $R$ inf $P$ & $\mathrm{~N} / \mathrm{A}$ \\
\hline 7 & 21 & $\mathrm{~F}$ & 6 & $\mathrm{~L} C \mathrm{CP}(\mathrm{CB})$ & $\begin{array}{l}\text { LEV OXC,PHB, } \\
\text { TPX }\end{array}$ & L CP & $\mathrm{L} C P$ & LP & LC HM & R P & $L \inf P$ & FCD2b \\
\hline 8 & 11 & M & 10 & L TPO (cyst) & STM,VPA & L FT & LFT & Bi FT & $\mathrm{N} / \mathrm{A}$ & $\mathrm{N} / \mathrm{A}$ & $\mathrm{N} / \mathrm{A}$ & $\mathrm{N} / \mathrm{A}$ \\
\hline 9 & 23 & M & 2 & $\mathrm{~L} \mathrm{~T}(\mathrm{CH})$ & CLB, LCS PHT & R TP & R P & Bi F & R HM & R FI & $\mathrm{R}$ inf $\mathrm{TO}$ & FCD1a \\
\hline 10 & 23 & $\mathrm{M}$ & 20 & FM (SOL) & CBZ, TPX VPA & R FT & R FT & $\mathrm{N} / \mathrm{A}$ & $\mathrm{N} / \mathrm{A}$ & $\mathrm{N} / \mathrm{A}$ & $\mathrm{N} / \mathrm{A}$ & $\mathrm{N} / \mathrm{A}$ \\
\hline
\end{tabular}

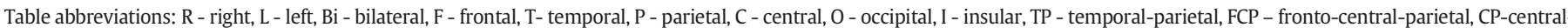

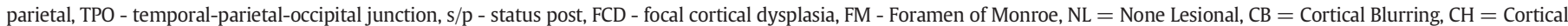

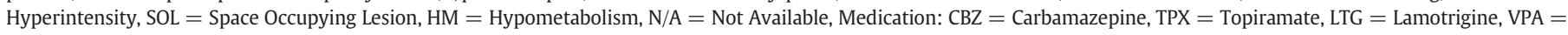
Valproic Acid, LEV = Levetiracetam, OXC $=$ Oxcarbazepine, CLB $=$ Clobazam, PHT $=$ Phenytoin, $\mathrm{LCS}=$ Lacosamide, PHB $=$ Phenobarbital, $\mathrm{STM}=\mathrm{Sulthiame}$.

bundles. In contrast, motion interference is influenced by cable orientation and, therefore, is expected to be more prominent in across-bundle measurements (sets 3 and 4). Since all motion interferences are distributed differently in the along-bundle and across-bundle measurements, daEEG allows suppression of both BCG and non-BCG interference in a strong magnetic field, without use of an additional device.

\section{Construction of the electrode array}

An MR compatible EEG cap equipped with standard $(5+5 \mathrm{k} \Omega) \mathrm{RF}$ shielding resistors and with 64 electrodes at standard 10-10 electrode positions (Brain Products $\mathrm{GmbH}$, Gilching, Germany) was adopted. 32 electrodes were kept in their original location, while the other 32

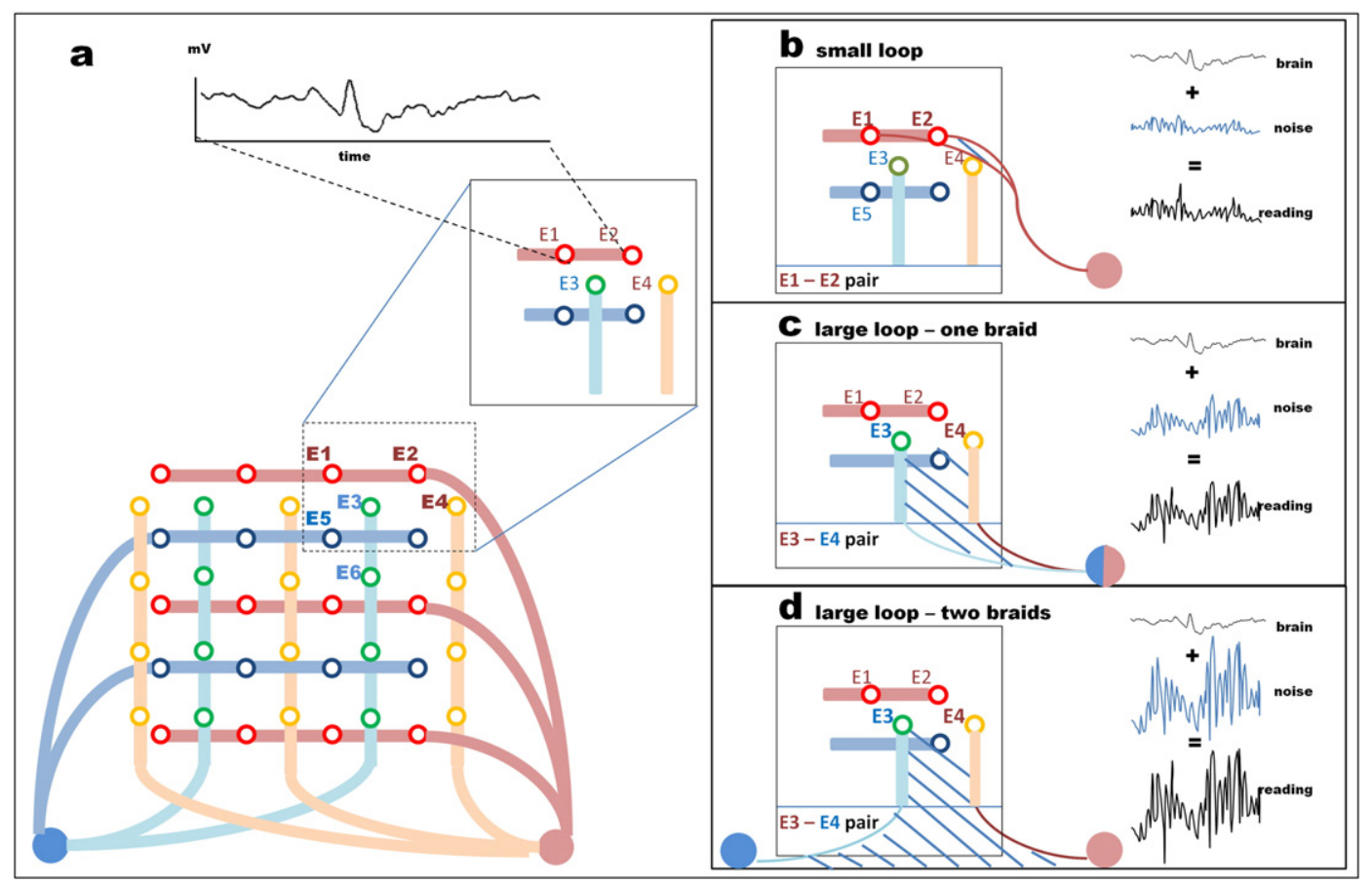

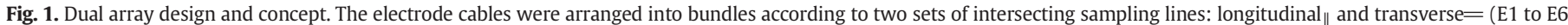

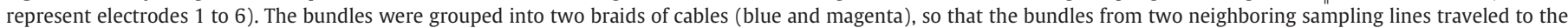

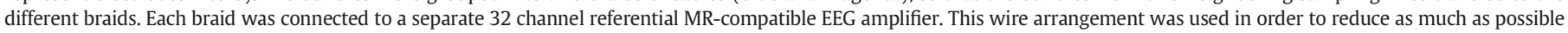

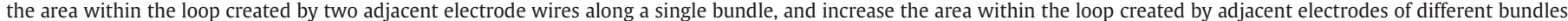

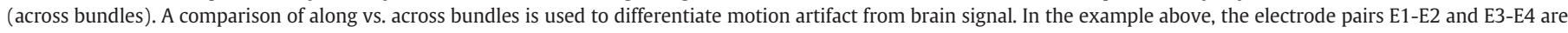

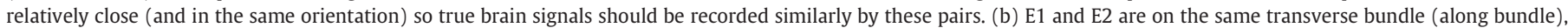

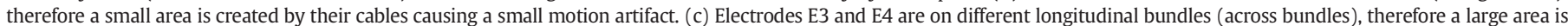

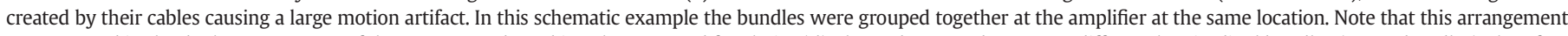

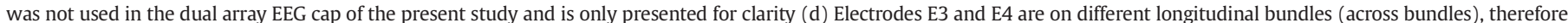

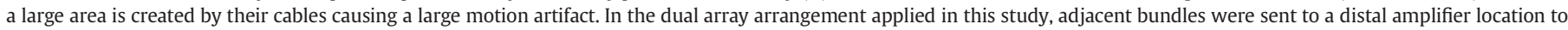

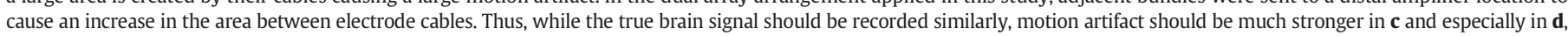
allowing separation between signal and noise. 


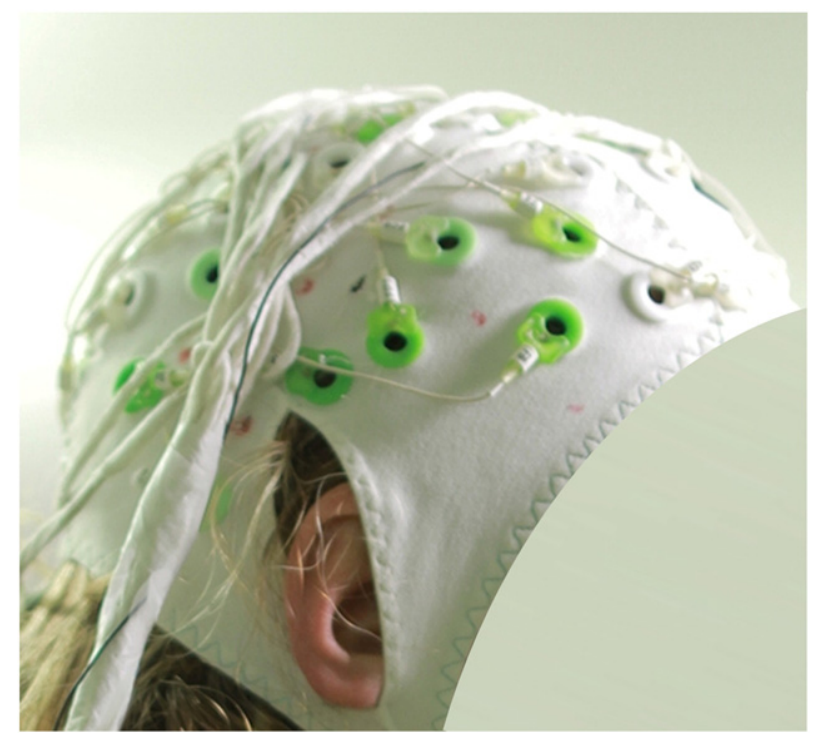

Fig. 2. A photograph of the daEEG cap used for this research.

electrodes were shifted two to three centimeters anterior left to the original electrode locations. As with the standard Brain Products cap, the reference and ground electrodes were connected to the midanterior part of the scalp midline. The leads of the reference and ground electrodes were split in two, so that these electrodes were connected to both amplifiers: only one reference and one ground electrode were used for each amplifier (as with the 64 Brain Products Cap). An additional electrode was placed on the patient's back as a reference ECG measurement. The wires from each of the 32 channel group of electrodes were connected to a standard referential MR-compatible EEG amplifier (Brain Products, Gilching, Germany). The electrode wires were arranged into bundles according to two sets of intersecting sampling lines: longitudinal and transverse as previously described. The bundles were grouped into two braids of cables each connected to a different amplifier. Bundles from two neighboring parallel sampling lines traveled to the different braids. The electrodes, bundles and braids were fixed to the EEG cap by leucoplast plaster. Finally, the braids were turned rostrally to their connectors and the cap itself was further secured to the occipital and anterior regions by leucoplast. A photograph of the version of the daEEG array used in the present study (Version 1) is shown in Fig. 2. Other variations of the daEEG cap, with standard electrode positions are described in the Supplementary material (Supplementary Fig. S6).

\section{EEG acquisition}

EEG was recorded both outside and inside the MRI scanner. Electrically conductive Abralyt 2000 gel (EasyCap GmbH, Herrsching, Germany) was used.

\section{fMRI acquisition}

MRI scans were performed in a 3.0 T MRI scanner (GE Signa EXCITE, Milwaukee, WI, USA) using a body transmitter coil and an eight channel head receiver coil. The EEG-fMRI recordings were performed in $20 \mathrm{~min}$ sessions of scanning; 3-5 such sessions were recorded during each patient scan. Patients were instructed to lie still and remain at rest. The helium pump was on during the recording as well as air conditioning inside the bore. A T2* - weighted, gradient echo, echo planar imaging (EPI) sequence was used for recording the fMRI images (TR/TE/flip angle: 3000/35/90). Thirty nine axial slices (thickness/gap: 3/0) were collected (FOV: $22 \times 22 \mathrm{~cm}$; matrix size: $128 \times 128$ ). In addition, a high resolution T1-weighted 3D $(1 \mathrm{~mm} \times 1 \mathrm{~mm} \times 1 \mathrm{~mm})$ volume was obtained using spoiled gradient echo (SPGR) sequence.

\section{fMRI image analysis}

The data analysis was performed using SPM5 software (http://www. fil.ion.ucl.ac.uk/spm). Preprocessing included slice timing correction, 3D motion correction and co-registration to the anatomical image. No normalization to an anatomical atlas was performed. The data was smoothed spatially with an $8 \mathrm{~mm}$ full width at half maximum (FWHM) Gaussian kernel. For each 20 min session, the first six functional volumes were excluded from analysis. Functional EPI data were automatically aligned and co-registered with 3D anatomical data and manually corrected if necessary. Standard SPM event related fMRI analysis was performed using a general linear model, using the timing of the detected EEG epileptiform waveforms (spikes or rhythms) as events. To account for variability in hemodynamic response and to follow the dynamic of epileptic event, predictors were shifted by lags of $-9,-6$, $-3,0,3,6$ and 9 s (Kobayashi et al., 2006).

\section{Artifact suppression: implementation of the daEEG concept}

The EEG data analysis was performed using EEGLAB software. After gradient interference suppression (FMRIB plug-in for EEGLAB, provided by the University of Oxford Centre for Functional MRI of the Brain), down-sampling from $5000 \mathrm{~Hz}$ to $250 \mathrm{~Hz}$ and band-pass filtering 0.5$40 \mathrm{~Hz}$, the referential montage measurements were recalculated into virtual bipolar measurements both along bundles (minimizing movement interference) and across bundles (maximizing interference). This resulted in four sets of bipolar measurements as previously described. The virtual bipolar array was decomposed using the logistic infomax independent component analysis (ICA) algorithm (Bell and Sejnowski, 1995; Delorme and Makeig, 2004). ICA was applied to the EEG data in order to identify and separate components which are differently distributed between the channels. The effect of motion artifacts is larger in data corresponding to measurements between electrodes connected to different bundles (across bundles) in comparison to electrodes connected to the same bundle (along bundles) (due to a larger wire loop). The ICA components with substantially higher weight sums in across montages compared to along montages are considered as artifact-affected components and are removed.

The ICA components were classified to noise or signal components according to three criteria:

1. Degree of two-dimensional correlation between ICA coefficients' (weights) spatial distribution along bundles and across bundles. The spatial distribution maps of each component at each of the four electrode pair groups (along longitudinal, across longitudinal, along transverse and across transverse) was maintained using topoplot function (from EEGLAB). All four types of bipolar EEG measurements (overall 85 bipolar measurements) were inserted into one ICA. The ICA produced independent components, each associated with a vector of 85 weights representing the spatial distribution of these components. Entries 1-24 of this vector correspond to along-transverse (Al-Ta) measurements, i.e. these are the weights of components on along-transverse bipolar channels, Entries 25-43 to along longitudinal (Al-Lo), entries 44-62 to across transverse Ac-Ta and entries 6385 to across longitudinal (Ac-Lo). The four maps in Fig. 3b, c and d correspond to the topographical plots created by these four sections of the weight vector. Finally, the spatial correlation was calculated between the first and fourth entry groups (Al-Ta vs. Ac-Lo) and between the second and third entry groups (Al-Lo vs. Ac-Ta). After performing topographical mapping of each of these four unevenly long vector sections, four similar size matrices were created by topographically interpolating the ICA weight topography for each of these four entry groups to yield the same (virtual) electrodes for 2D correlation. The correlation between spatial distribution of ICA coefficients of along longitudinal pairs and spatial distribution of ICA coefficients of across transverse pairs was calculated using corr2 MATLAB 


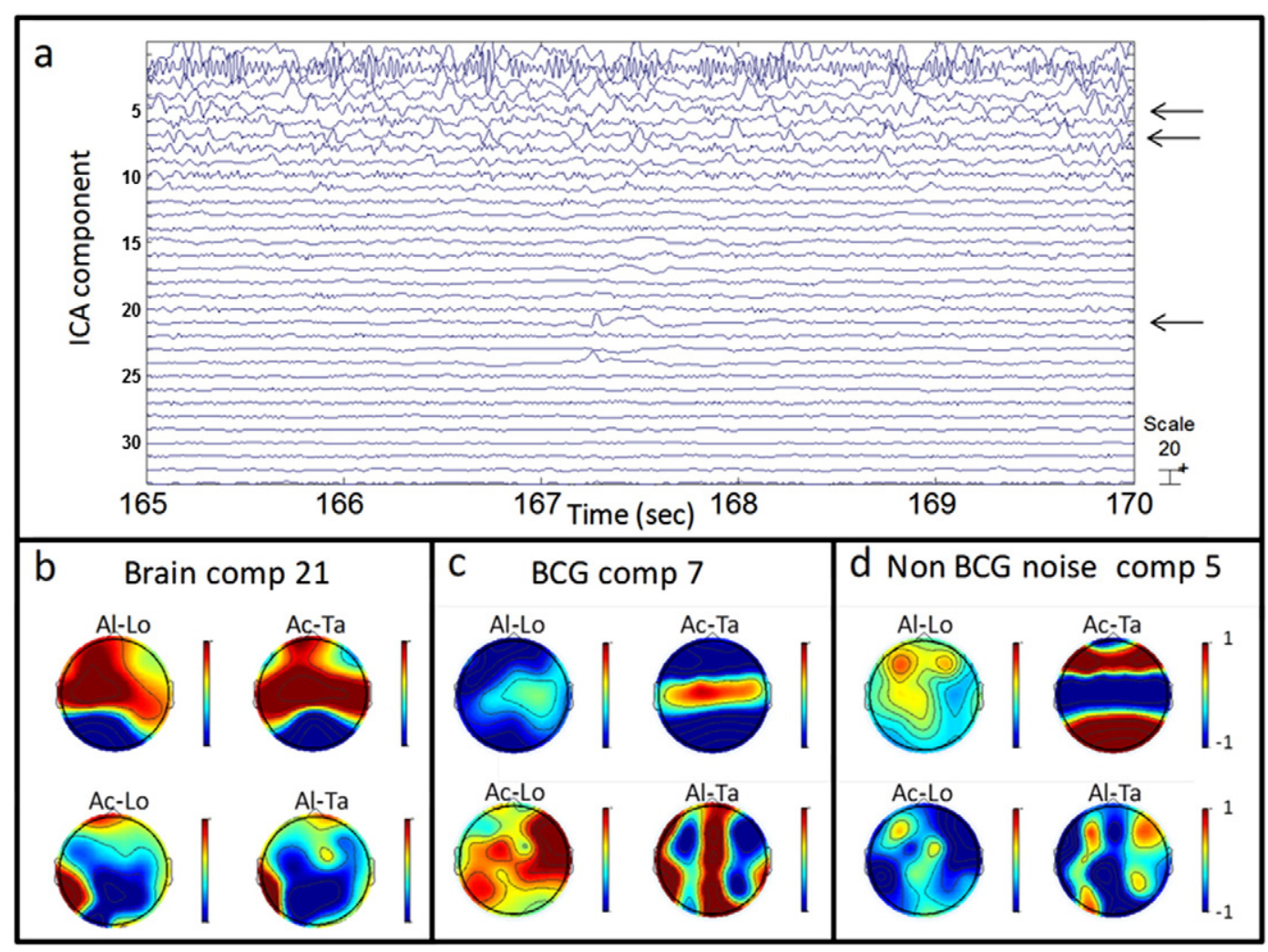

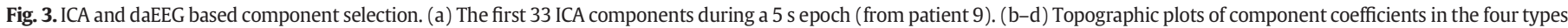

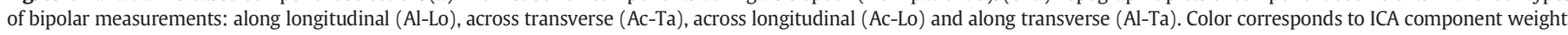

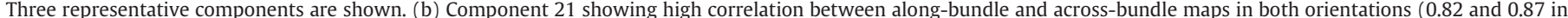

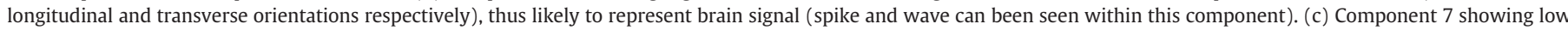

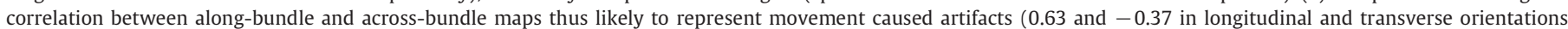

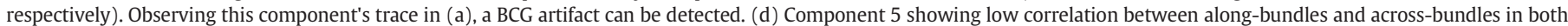

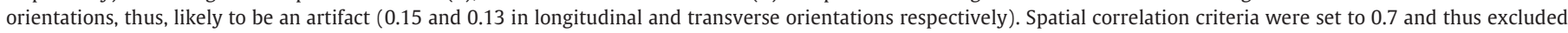
components 5 and 7 from the analysis and retained component 21. The four maps in b, c and d correspond to four parts of independent component weights vector (see text).

function (as both these groups are in the same longitudinal orientation and thus should show similar representation for brain signals). Similarly, the spatial correlation of along transverse oriented pairs and across longitudinal pairs was calculated (as both these groups are in the same transverse orientation and thus should show similar representation for brain signals). The maximum correlation value of the two (longitudinal orientation and transverse orientation) was taken. Based on the assumption that the spatial distribution of real neuronal signals does not change with different wiring while movement interference does, components with low along-across spatial correlation were removed from the data. See Fig. 3 for an example. Cutoff was set to $0.65 \pm 0.05$.

2. Amplitude ratio between ICA coefficients of along and across bundles (again performed separately for each orientation and the maximum taken). Assuming that different wiring will not affect the coefficient amplitude of real neuronal signals but will affect movement interference, the components with extreme along/across ratio were removed from the data. Cutoff ranged from 2 to 4 .

3. Degree of time correlation to ECG time course measured with ECG electrode (for the removal of BCG artifacts). To quantify the degree to which ICA components contain cardiomechanical artifacts, we calculated the temporal correlation with ECG in the following way: We first detected the timing of the R peaks (of the QRS complex) in the ECG signal using the FMRIB toolbox. Next, for each component, windows of $450 \mathrm{~ms}$ length were created around each R event ( 50 ms prior and 400 ms post R peak) and Pearson correlations between these $\mathrm{N}$ windowed time-series were averaged, producing a mean over [ $\mathrm{N}$ x ( $\mathrm{N}$ - 1)/2 comparisons], component specific, ECG based correlation value. If the component was not affected by the cardiomechanical movement, it should not be time locked to it and thus the calculated mean correlation should be low (as different QRS instances would be uncorrelated). The advantage of this calculation, over a simple correlation between the component and the ECG signal is that it does not assume that the cardiomechanical artifact in the EEG resembles the waveform of the ECG signal (See Fig. 4 for an example).

The components with high correlation to ECG were removed. Cutoff was set to $0.3 \pm 0.1$ (If applying Fisher $\mathrm{Z}$ transformation to the correlation values, the thresholds should be slightly increased, see Supplementary material).

Threshold selection: The thresholds for all three criteria were chosen subjectively based on the inspection of the reconstructed EEG traces. The EEG traces were reconstructed iteratively changing the thresholds' cutoff. In the first three patients the thresholds were not limited and the iterations were taken through the whole range of spatial and ECG (temporal) correlations $0-1$; the iterations of amplitude ratio were taken from 1 to 4 . At this stage the authors observed that favorable data quality can be achieved within a relatively narrow threshold range: 0.6 to 0.7 for spatial correlation, 0.2 to 0.5 for temporal correlation with ECG and 2 to 4 for the amplitude ratio threshold. It was, however, impossible to completely define fixed thresholds for all patients or even for different sessions of one patient. Setting stricter thresholds outside these ranges (such as setting a spatial correlation threshold above 0.7 or an amplitude threshold below 2 , thus removing more independent components) typically led to "over cleaned" data and setting weaker thresholds outside these ranges - to noisy data. Therefore, in the remaining seven patients the threshold iterations were performed 
a

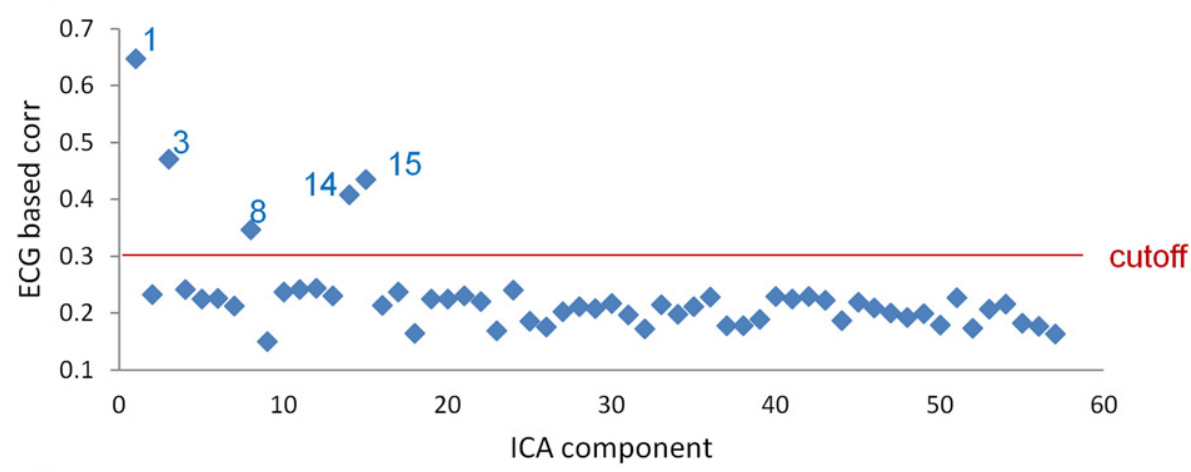

b
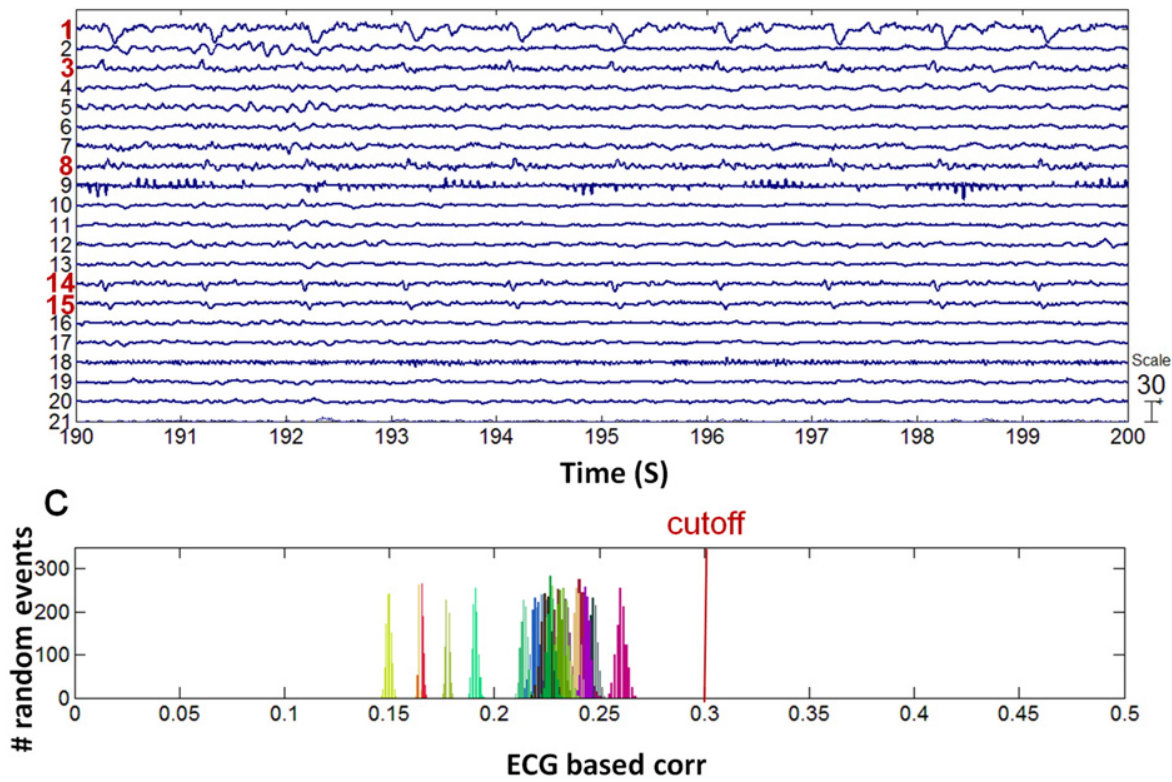

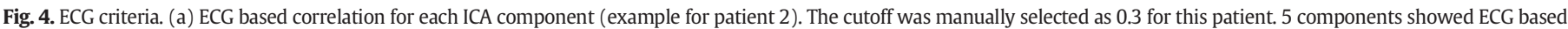

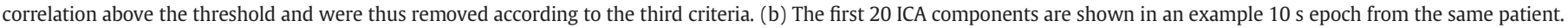

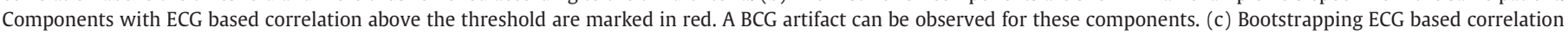

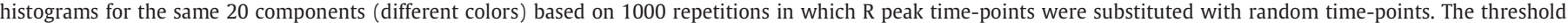
correlation chosen was above all random iterations $(20 \times 1000)$.

within the above mentioned narrow limits. Again, the final threshold set was defined subjectively by visual inspection of the data. At this stage the threshold iterations were generally done in the following way: first the temporal correlation with ECG threshold was chosen iterating three values: $0.2,0.3$ and 0.4 and inspecting the three resultant denoised EEG traces (For one patient it was necessary to extend this range and use a cutoff of 0.5 ); then, using the chosen ECG correlation threshold, the spatial correlation and amplitude ratio were chosen by three iterations for each criteria ( $0.6,0.65$ and 0.7 for spatial correlation and 2, 3 and 4 for amplitude ratio), resulting in an inspection of nine denoised EEG traces. Overall twelve EEG traces were inspected in the process of criteria set threshold selection.

To deal with non-stationarity of ICA random matrix initialization, we applied ICA decomposition ten times (EEGLAB uses a different starting point with every new ICA). The criteria set for component selection were defined according to the first ICA. The selection of components was done separately for each ICA; and the components, which passed the criteria set, were transformed back into EEG channel space. Thus, ten de-noised EEG traces were constructed. Lastly, these ten EEG traces were averaged to create an average de-noised EEG trace. Five ICAs were sufficient to achieve a stabilized result and additional ICAs beyond $\mathrm{N}=$ 5 provided negligible improvements (Supplementary material Fig. S5).
Ten repetitions of ICA provides good safety margin regarding the ICA stability.

\section{EEG evaluation}

Detection of epileptiform activity was performed manually by a neurologist experienced in EEG interpretation (last author, MM). The epileptic or non-specific abnormal waveforms were manually classified into types (spike and wave complexes, epileptic rhythms and nonrhythmic slow waveforms) and their onset time and durations were recorded. The timings of events for every waveform type were used as a predictor in an fMRI event related analysis. In addition, epochs concurrent with large EEG artifacts were excluded from the fMRI analysis.

\section{Validation of EEG data quality}

EEG data quality was compared between the proposed framework for noise reduction and a standard framework for gradient and BCG artifact removal as implemented in FMRIB. For this purpose, after gradient artifact removal, data of all subjects underwent Cardio-ballistic artifacts removal using the FMRIB Optimal Basis Set (OBS) approach. The dataset was also down-sampled to $250 \mathrm{~Hz}$ and band-pass filtered to $0.5-40 \mathrm{~Hz}$. 
The Signal-to-noise ratio (SNR) of the detected EEG events was compared between both approaches.

\section{SNR analysis}

For evaluating the SNR we chose a measure aimed at representing spike detectability (SNR detectability, see Eq. 1). Commonly, the SNR of externally triggered ERP data is assessed by defining the signal as the average of peak-to-peak data (this should also average out background brain activity); the noise is then defined as the standard deviation of pre-stimulus epoch. In the case of non-averaged epileptic spikes, background brain activity is not averaged out and it is unclear what proportion of brain signal is intermixed with interference during the pre-spike interval. We assumed that spike detectability mostly results from the relationship between peak-to-peak amplitude of the spike and the standard deviation of the same epoch. Thus, we used a modified approach comparing the peak-to-peak spike amplitude to standard deviation during the same epoch. Each epoch was defined as $1 \mathrm{~s}$ long, starting $0.5 \mathrm{~s}$ before spike maxima, and was extracted separately for each noise reduction method (daEEG vs. OBS). Notice that the entire epoch (including the spike itself) has been used. The resulting values were averaged across individual spikes and channels for each subject. This mean SNR measure was compared between the two noise reduction methods for each subject using a paired $t$-test.

$S N R_{\text {detectability }}=\left(x_{\max }-x_{\min }\right) / \sqrt{\frac{\sum_{t=1}^{n}\left(x_{t}-\mu\right)^{2}}{n}}$

Number and amplitude of detected spikes after daEEG and OBS noise reduction

In order to test whether differences in noise reduction between daEEG and OBS are translated into better spike detection we performed following analysis. In addition to the manual detection of epileptic spikes in the EEG traces de-noised by daEEG approach, the same neurologist (MM), blind to the labeling of subjects, detected spikes in the EEG traces de-noised by OBS. At least one year passed between daEEG and OBS spike detection. The patients' data were recorded in several 20minute sessions. Spike detection following OBS noise reduction was performed on the 20 min recording with the maximal number of spikes (as detected and quantified following daEEG noise reduction). The number of detected spikes after daEEG and OBS was compared using Wilcoxon signed rank test. Another question was whether or not the difference in noise reduction leads to difference in the detectability of small spikes. In order to test this, the amplitudes of spikes detected after daEEG noise reduction but not after OBS noise reduction were compared to amplitudes of spikes detected after both noise reduction methods. As a test for significant differences unpaired Student $t$-test was applied to the results of every patient.

\section{Hybrid EEG trace analysis}

To evaluate spike detection sensitivity and specificity and to ensure that daEEG does not eliminate additional, nondetected, epileptic transients (or any brain originated activity), an analysis in which the timing of the events is known prior to noise reduction procedure is necessary. Thus, we constructed a hybrid EEG trace, where epileptic spikes from out-of-scanner EEG traces were inserted into the in-scanner EEG trace. This was performed by adding one second spike-containing epochs from the out-of-scanner recording in random temporal locations within the in-scanner recording. Subsequently, daEEG ICA noise reduction procedure was performed (Fig. 5). The peak-to-peak and SNR of these events was calculated as before, once in their original out-of-scanner locations and once in in-scanner locations after ICA noise reduction. In addition we calculated peak-to-peak amplitude and SNR for hybrid trace de-noised by OBS.

Spike detection of the hybrid data was also performed, in which the number and timings of spikes that should be detected is known a-priori. Only four of the ten patients had spikes recorded out-of-scanner by the same array used in EEG-fMRI recordings. In order to compare the spike detectability in hybrid trace we constructed 80-second EEG traces containing epochs of spikes and background activity, in the following way: We inserted 40 segments (each of one-second length) of EEG traces recorded out-of-scanner into a 20 min long EEG recorded simultaneously with fMRI. Inserted segments consisted of 20 segments with spikes and 20 without. The out-of-scanner data was inserted into in-scanner data of the same patient. The resulting hybrid 20-minute EEG trace was denoised by daEEG and OBS approaches. After noise reduction, all 80 hybrid epochs (40 from daEEG de-noised trace and 40 from OBS denoised trace) were randomly connected "head-to-tail" into one 80-s long EEG trace. The resulting trace contains four categories of randomly placed one-second hybrid epochs (twenty epochs per category) containing: 1) spikes de-noised by daEEG; 2) spikes de-noised by OBS; 3) background (without spikes) de-noised by daEEG; 4) background (without spikes) de-noised by OBS. The neurologist manually analyzed this trace and marked every one-second epoch as containing or notcontaining a spike. We calculated the true and false positive rates and computed the sensitivity and specificity of spike detection for all four patients together using the following formulas:

Sensitivity = number of true positives/

(number of true positives + number of false negatives)

Specificity $=$ number of true negatives /

(number of true negatives + number of false positives)

\section{Results}

\section{daEEG interference suppression and SNR comparison}

For each patient daEEG noise reduction was compared to the OBS noise reduction. Fig. 6 presents an example of EEG traces before and after noise reduction procedures (patient 9) and Fig. 7 presents an additional example in which out of scanner epileptic waveforms can be compared to in-scanner waveforms following noise reduction (patient 7).

Only recordings that exhibit epileptic spike activity on the EEG were included in the analysis, resulting in a total of 21 sessions (from 10 patients). SNR of EEG processed with the proposed framework was significantly higher than with the OBS method $(11.05 \pm 3.10,8.25 \pm 1.01$ $p<0.00001$ paired $t$-test) representing a 30\% average improvement in SNR.

\section{Hybrid analysis}

Out-of-scanner interictal epileptiform activity was found in four of ten patients (Out-of-scanner recordings were usually shorter than inscanner ones and patients usually remained awake). For each of these four patients, 20 spikes from out-of-scanner recording were implanted at random temporal locations and daEEG noise reduction procedure was performed (Fig. 5). In order to rule out the possibility that the ICA step diminishes epileptic events, we calculated the peak-to-peak spike amplitude along with SNR. Peak-to-peak amplitude of out-of-scanner implanted events in the hybrid EEG traces after daEEG noise reduction were on average $1.17 \pm 0.19$ times the peak-to-peak amplitude of the same spikes in their original out-of-scanner form $(1.24 \pm 0.22,1.1 \pm$ $0.17,1.2 \pm 0.21,1.09 \pm 0.14$, for patients $2,3,7$, and 8 respectively). Thus, none of these patients demonstrated significantly decreased 
a

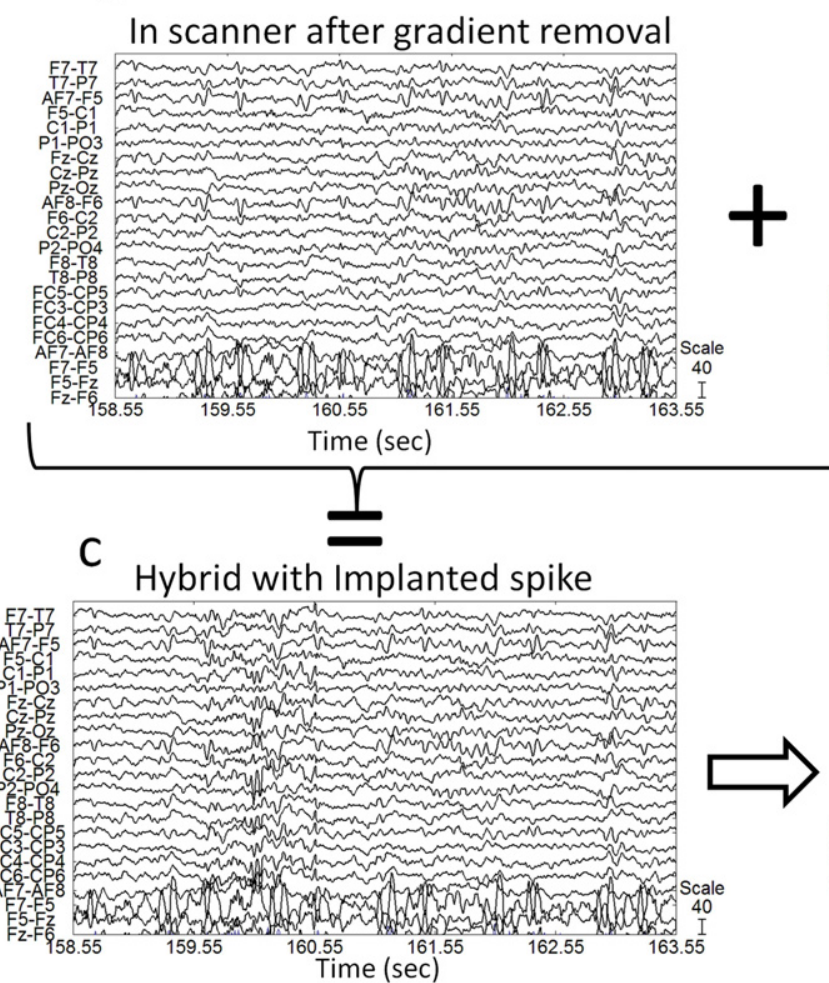

b

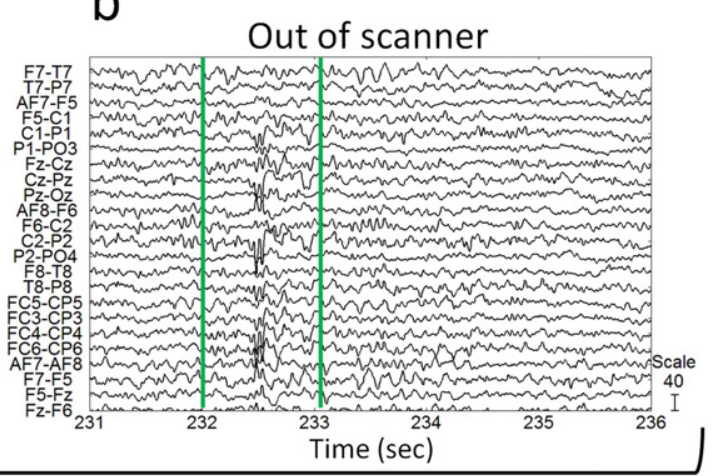

d

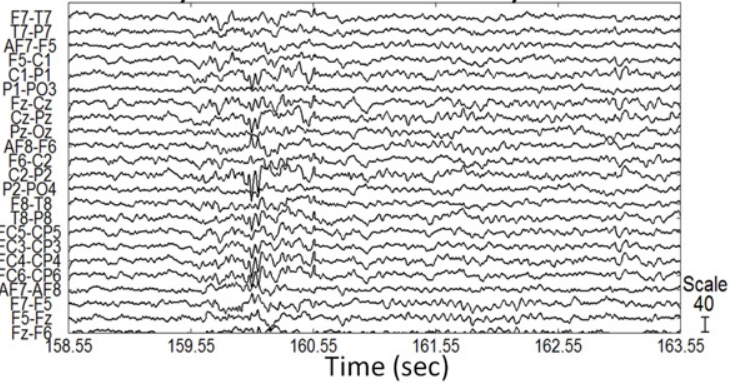

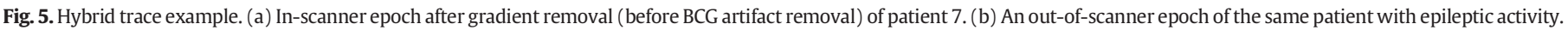

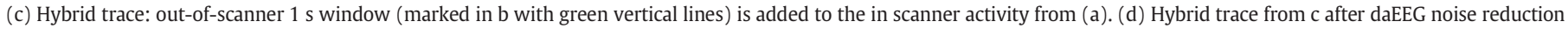
procedure.

implanted spike amplitude compared to their out-of-scanner form. SNR of implanted spikes was on average $0.81 \pm 0.14$ times the SNR of the same spikes in their original out-of-scanner form $(0.81 \pm 0.17$, $0.80 \pm 0.12,0.82 \pm 0.16,0.79 \pm 0.11$, for patients $2,3,7$ and 8 respectively). After OBS noise reduction the peak-to-peak amplitudes were $1.42 \pm 0.26$ times the peak-to-peak amplitude of the same spikes in their original out-of-scanner form $(1.44 \pm 0.22,1.45 \pm$
$0.17,1.25 \pm 0.21,1.52 \pm 0.14$, for patients $2,3,7$, and 8 respectively). After OBS noise reduction SNR of implanted spikes was on average $0.80 \pm 0.13$ times the SNR of the same spikes in their original outof-scanner form $(0.78 \pm 0.15,0.93 \pm 0.13,0.70 \pm 0.11,0.77 \pm$ 0.12 , for patients $2,3,7$ and 8 respectively). See Supplementary Fig. S1 for a comparison between daEEG and OBS noise reduction of hybrid traces.

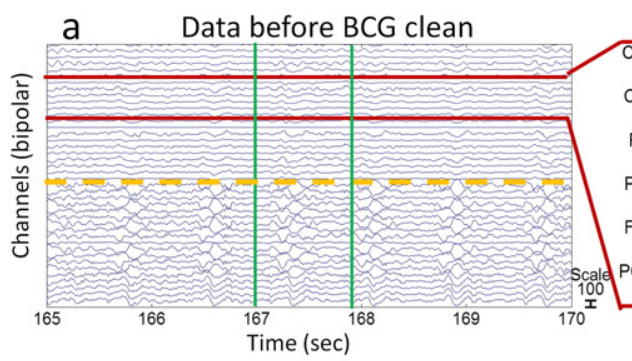

b Before BCG clean zoom
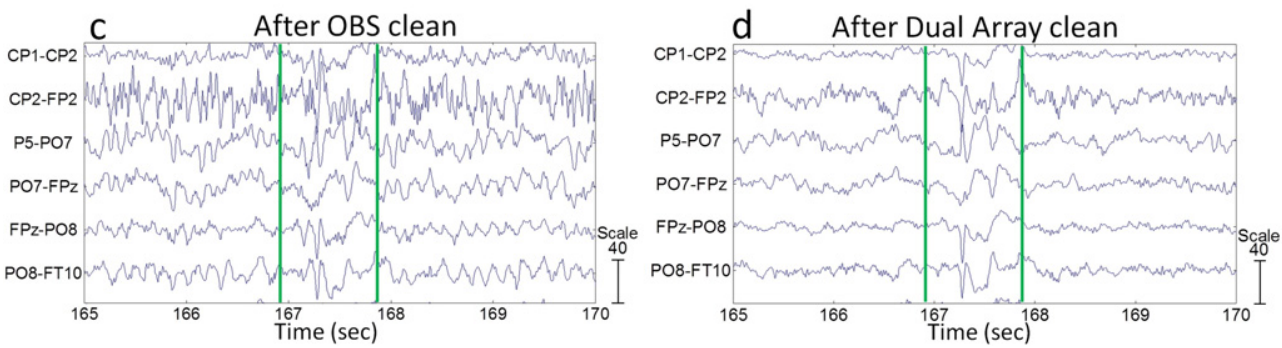

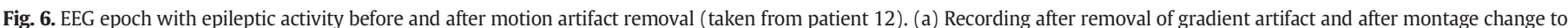

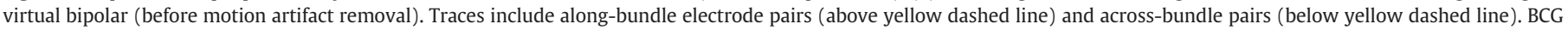

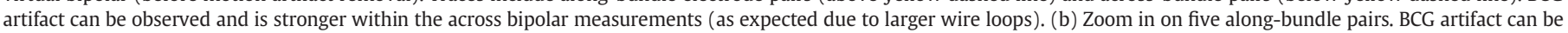
observed. (c) Data after standard BCG removal (EEGLAB software). (d) Data after dual array motion artifact removal. Spike and wave can be seen within the green vertical lines. 

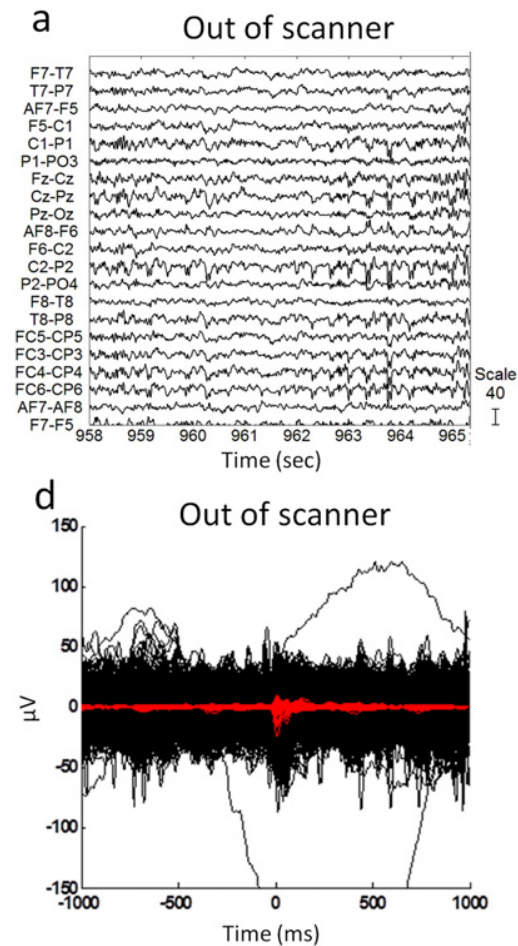

b

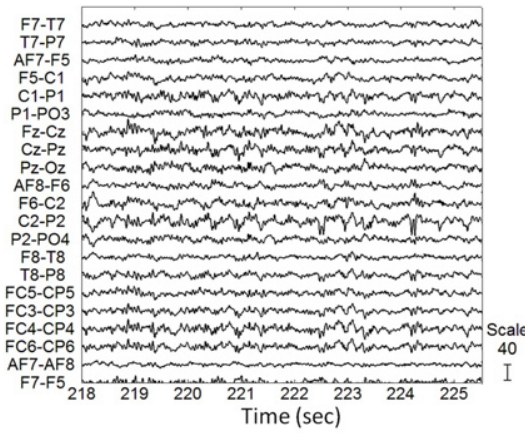

e

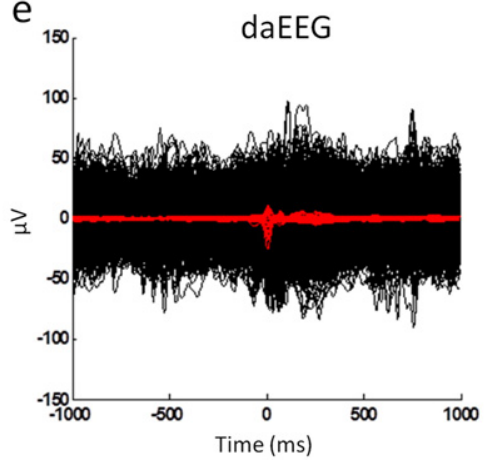

C OBS

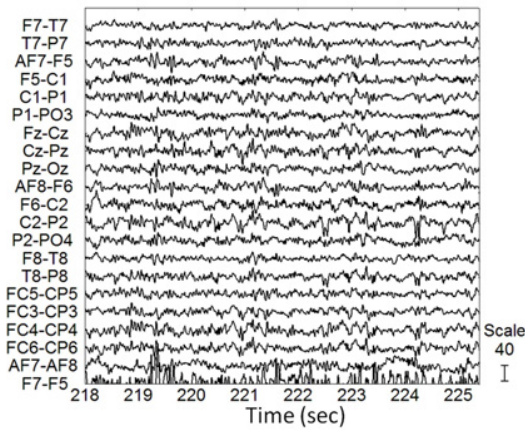

$f$

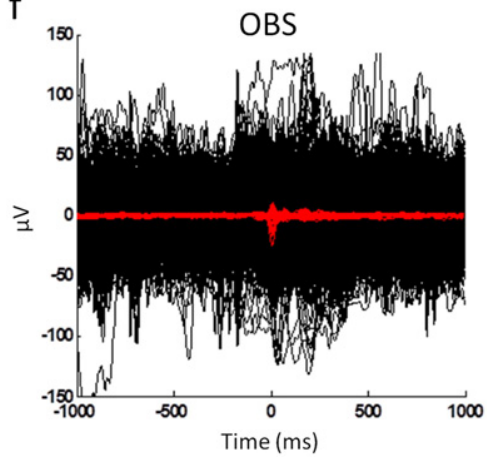

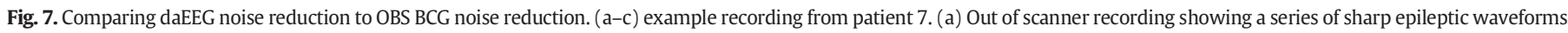

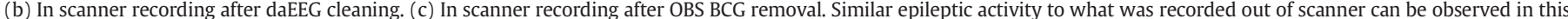

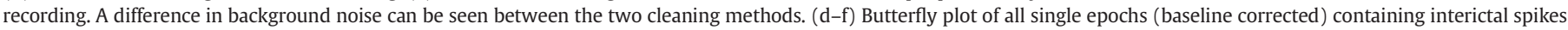

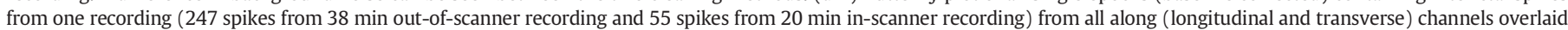

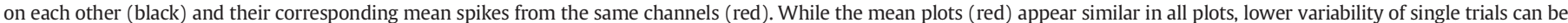
observed following daEEG compared to OBS, implying lower background noise and higher SNR.

\section{BCG artifact}

In some patients we identified two types of BCG components separated by ICA (Fig. 8). One type had low spatial correlation between along- and across-bundles (Fig. 8b) measurements and another type had high spatial correlation (Fig. 8a).
Number and amplitude of detected spikes after daEEG and OBS noise reduction

In nine out of ten patients the number of spikes detected after daEEG noise reduction was higher than after OBS noise reduction (Fig. S3, Supplementary material). In one patient the number of detected spikes was a
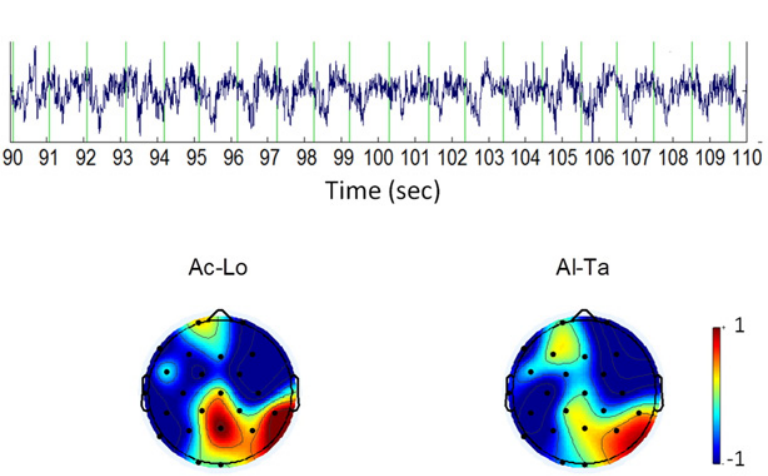

Spatial correlation $=0.9$ b
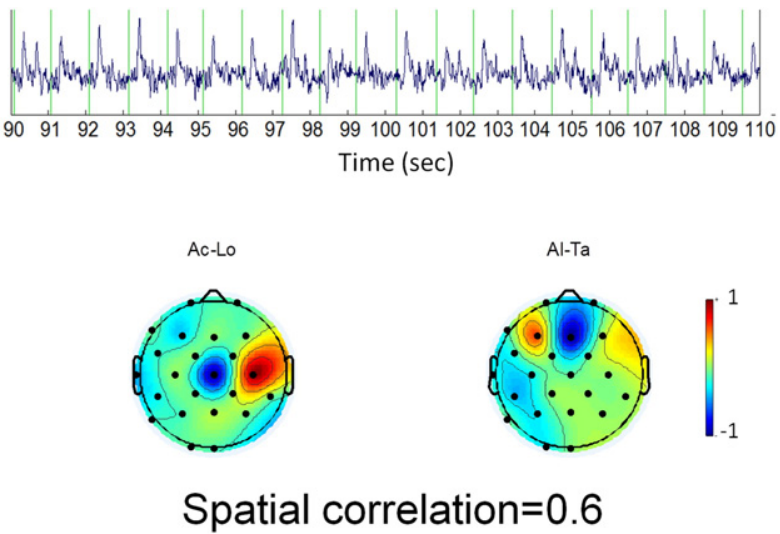

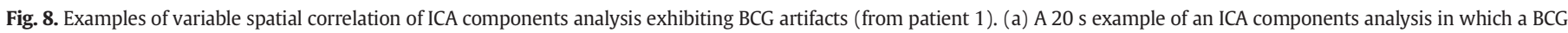

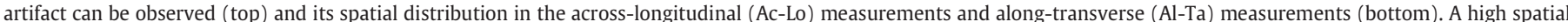

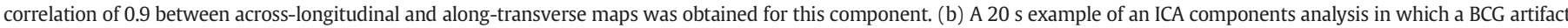

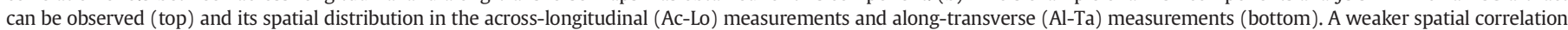
of 0.6 between across longitudinal and along transverse maps was obtained. 
higher after OBS noise reduction. A mean of $56 \pm 60$ and $23 \pm 18$ spikes were detected for daEEG and OBS respectively. A Wilcoxon signed rank test revealed significantly more spikes detected after daEEG noise reduction compared to after OBS noise reduction $[Z=2.5, p<0.05]$.

In seven out of ten patients the spikes detected after daEEG noise reduction but not after OBS noise reduction had significantly lower amplitudes than the spikes detected after both noise reduction methods. In three patients no significant difference was found (Fig. S3 of the Supplementary material). On average the peak-to-peak amplitude of spikes detected following both noise reduction methods was $1.11 \pm 0.1$ higher than the peak-to-peak amplitude of spikes detected following daEEG noise reduction only.

Hybrid trace spikes were detected with a mean sensitivity of 0.58 and 0.46 following daEEG and OBS noise reduction respectively and a mean specificity of 0.94 and 0.80 following daEEG and OBS noise reduction respectively (Table 2 ).

\section{Case reports}

fMRI statistical maps, created as the final step of daEEG were considered as experimental results in the patients' pre-surgical workup. Four of the ten patients included in this report were further evaluated using invasive procedures. In these cases, the epileptogenic zone suggested according to the daEEG analysis was included as a target for invasive study.

In patient 5 ( $13 \mathrm{y} / 0$ male), 50 spike-and-wave complexes were found in an 80 min recording. BOLD activation with the predictor set to these events at a lag of $6 \mathrm{~s}$ suggested a strong activation cluster at the right superior frontal sulcus. MRI suggested focal cortical dysplasia within this sulcus. This region was resected and the patient has been seizure free post operatively for one year. Pathological examination verified cortical dysplasia at the specified location. (Fig. S4 in Supplementary material).

In patient 6 ( $15 \mathrm{y} / 0$ female), 51 spike-and-wave complexes were found in a $60 \mathrm{~min}$ recording. BOLD activation with the predictor set to these events at a lag of $6 \mathrm{~s}$ suggested a strong activation cluster at a parietal/perisylvian location. This patient was implanted with a $7 \times 8$ fronto-parietal grid and one $1 \times 8$ temporal strip in the right hemisphere. Seizure onset was localized to 10 parieto-perisylvian channels (Fig. 9) and this region was resected. This patient has been seizure free post operatively for 1.5 years.

In patient 7 ( $20 \mathrm{y} / 0$ female), 54 spike/polyspike and wave complexes were identified in a 20 min recording. BOLD activation with the predictor set to these events at a lag of $6 \mathrm{~s}$ suggested a midsagittal superior frontal cluster. At a lag of $-6 \mathrm{~s}$ the suggested activation cluster was in the dorso-lateral aspect of the left parietal lobe. This patient was implanted with a $8 \times 6$ fronto-parietal grid and a $1 \times 8$ superior frontal strip on the left hemisphere. Seizure onset was found in 8 postcentral electrodes. This region was resected and the patient has been seizure free for two years. Pathological examination verified cortical dysplasia at the specified location. (Fig. S4 in Supplementary material).

In patient 9 ( $17 \mathrm{y} / \mathrm{o}$ male), 102 spike-and-wave complexes were detected in a 20 min recording. BOLD activation with the predictor set to these events at a lag of $6 \mathrm{~s}$ suggested three main activation clusters within the right hemisphere (Fig. S4 in Supplementary material): a premotor frontal cluster, a parietal cluster and a ventral occipitaltemporal cluster. This patient was implanted with an $8 \times 10$ frontotemporo-parietal grid and 3 occipital $1 \times 8$ strips. Electrodes at these three regions showed epileptic activity at seizure onset. More specifically, the occipital and frontal regions preceded the parietal region in seizure initiation. Considering these findings in conjunction with an MRI finding of a focal cortical dysplasia (FCD) in the occipital region, the occipital region was chosen for resection. Pathological examination verified cortical dysplasia at this location. Seizure frequency however, was not reduced post operatively.

\section{Discussion}

Artifacts generated by head motions inside a high magnetic field degrade the quality of EEG recordings and therefore decreases the chances of detecting transient events (Allen et al., 1998; Bonmassar et al., 2002). Some of these head motions, such as cardioballistic movements, have a relatively predictable effect on the EEG, albeit with large variability (Allen et al., 1998), whereas other non-BCG artifacts - such as those caused by the helium pump, floor vibrations and small head jerks are less studied. daEEG is a novel method developed for removing both BCG and non-BCG motion artifacts. In a group of ten patients with epilepsy we evaluated the method's ability to suppress motion artifacts while maintaining neurophysiological EEG morphology, thus allowing the detection of epileptic activity. We compared daEEG, which was analyzed semi-automatically, to automatic artifact removal with OBS, a commonly used approach (Niazy et al., 2005). daEEG allowed on average $30 \%$ better improvement in SNR of epileptic events in comparison to OBS. Examples of the comparison between daEEG and OBS can be seen in Figs. 6 and 7 (and in Supplementary Figs. S1 and S3). While the mean signal curves are similar to OBS, daEEG and out-ofscanner recordings, the individual event curves demonstrate a higher variance for OBS in comparison to daEEG. This example demonstrates that noise reduction achieved by daEEG was not at the expense of brain signal suppression, as this would have decreased the amplitudes of the mean curves.

Improved SNR may be particularly important when searching for transient potentials in the EEG data, such as in the case of epileptic spike detection. Indeed more spikes were detected after daEEG noise reduction compared to OBS in nine of ten patients. However, without knowing the true timing of these events we cannot know how many of these spikes were true positives, and how many spikes were still missed (false negative). Hence, we cannot calculate the spike detection sensitivity and specificity following daEEG or OBS. To deal with this issue we implanted known spikes from the relatively clean out-ofscanner recordings into the noisy in-scanner recordings resulting in a hybrid trace. This analysis revealed that both sensitivity and specificity were higher following daEEG noise reduction than after OBS (Table 2). In addition, hybrid trace analysis showed that SNR for the implanted spikes was higher after daEEG, compared to OBS noise reduction (Supplementary Fig. S1).

An evident question is whether this improved SNR and higher sensitivity and specificity would allow the detection of lower amplitude events that could not be detected following OBS. Our results suggest that this assumption is correct. For seven patients the amplitude of the spikes detected after daEEG noise reduction and missed following OBS had significantly lower amplitude compared to those detected by both methods (Fig. S3 in Supplementary material). On average, the difference in spike amplitude was only $11 \%$ (relatively low in comparison to the $30 \%$ SNR improvement). Nevertheless, this difference depends not only on SNR but on other factors such as the spike amplitude variance in the recording and the noise variance between epochs. For example, if spike amplitudes have low variability, and the noise varies highly

Table 2

Hybrid trace spike detectability in four patients (80 epochs with spikes / 80 epochs without spikes).

\begin{tabular}{|c|c|c|c|c|c|c|}
\hline Noise reduction method & Specificity & Sensitivity & False negatives & False positives & True negatives & True positives \\
\hline daEEG & 0.94 & 0.58 & 34 & 5 & 75 & 46 \\
\hline OBS & 0.80 & 0.46 & 53 & 16 & 64 & 37 \\
\hline
\end{tabular}


a
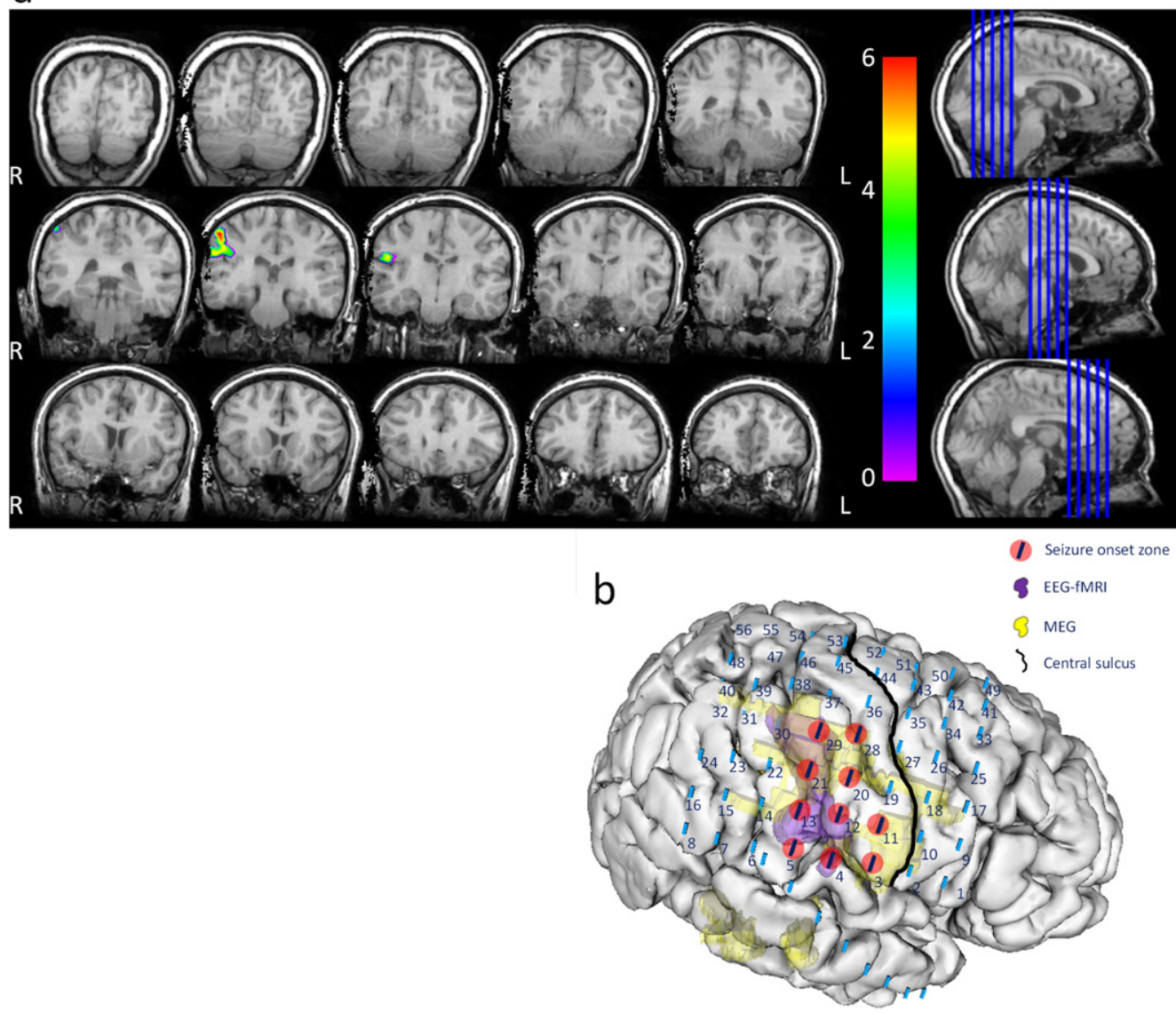

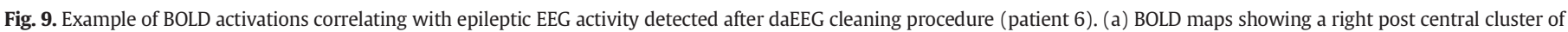

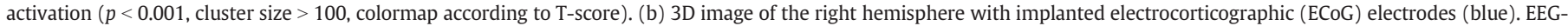

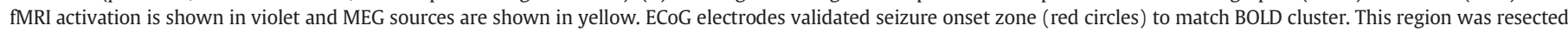
and the patient is seizure free for 2 years. See Supplementary material Fig. S4 for remaining cases with intracranial validation.

between epochs, the noise variability (and not spike amplitude) could be the main factor determining whether a given event will be detected or not.

The results described above compared daEEG with OBS, a method that was designed to remove gradient and BCG artifacts (Niazy et al., 2005). In OBS, a template is created using the selected basis set and subtracted from each cardioballistic event. This method was not designed to remove non-BCG movement artifacts. daEEG on the other hand suppresses both BCG and non-BCG artifacts. Other solutions for removing non-BCG artifact have been developed (Bonmassar et al., 2002; Chowdhury et al., 2014; Jorge et al., 2015; LeVan et al., 2013; Masterton et al., 2007). Common to these approaches is the use of reference motion sensors, which do not record brain signals. These approaches have the advantage of obtaining a "pure" assessment of the motion artifacts. Additionally, some reference motion arrays do not require high electrode density or additional equipment (Jorge et al., 2015). The main limitation of using reference motion sensor methods is their limited efficacy in cases of non-rigid body motion artifacts. Such artifacts can appear due to slight movements of individual EEG cables and may not be fully reflected in the wires used as motion sensors. While most studies consider only rigid body movements when modeling for EEG artifacts, LeVan et al. (2013) observed a substantial non-linear aspect of the BCG artifact which they attributed to non-rigid body and head movements. Non-rigid-body movements of EEG leads, caused by rigid body head movements as well as scalp expansion, were mentioned as probable causes for this non-rigid body artifact. Hence, in an attempt to separate between the EEG artifacts and brain signals from a specific electrode, it may be beneficial to manipulate the amplitude of the motion artifact by adjusting a specific EEG wire loop. The daEEG approach takes into account the assumption that EEG motion artifacts vary with changing wire arrangements, while true physiological signals do not, thus daEEG utilizes both small (along bundle) and large (across bundle) wire loops. This principle allows for noise reduction using a selection of ICA components based on the comparison between coefficients of along and across bundle channels. As suggested here, we assume that using the EEG channels as motion sensors may have an additional value over rigid body movement sensors for artifact suppression, particularly as motion artifact contains substantial non-rigid body movements.

In addition to the bundling of wires and the comparison between along and across bundle measurements, a main principle of daEEG is that the cable bundles travel in two orthogonal directions: longitudinal and transverse. Taking into account that the head is a three-dimensional object, daEEG cables create a three-dimensional net. Motion in nearly any direction results in a change of magnetic flux, which is nonparallel to the surface of at least some of the cable loops, thereby inducing electric currents. Such motion related currents can be distinguished from brain signals by comparison of their amplitudes and spatial distribution between along- and across-bundles measurements. While a "pure" rotation around the $\mathrm{z}$ axis of $\mathrm{B} 0$ will not lead to a change of magnetic flux across the cable loops, such "pure" motion is unrealistic and practically all head or cable movements inside the MR-scanner are associated with electric current induction.

In addition to the use of this unique hardware, daEEG employs ICA analysis for data decomposition. Several studies have reported the use of ICA for noise reduction in EEG recorded simultaneously with fMRI 
(Mantini et al., 2007; Srivastava et al., 2005). ICA decomposes the data into independent components, which allows the separation of signals originating from different independent sources, such as brain neuronal activity and motion artifact. How to distinguish between data components and noisy components that should be removed, is an open issue and varies between different research groups. Correlations with reference signals (ECG and/or EOG) was used by (Debener et al., 2008; Mantini et al., 2007; Srivastava et al., 2005). In addition, Debener et al. (2008) employed variance based analyses. Manual selection was performed by Mantini et al. (2007) and Huiskamp (2006). Vanderperren et al. (2010) employed more complex criteria set for component selection, which was based on several parameters: correlation, frequency content, autocorrelation, variance and peak-to-peak amplitude of reconstructed sources. However, daEEG allows utilization of a simple principle for independent component selection: components whose spatial distribution is independent of EEG cable arrangements correspond with brain activity, while those that are influenced by EEG cable arrangements correspond with motion artifacts (either BCG or non-BCG). Thus our method offers a new technique for ICA component selection.

Using the comparison of along and across bundles, signal amplitudes and spatial maps provide a good separation between signal and motion artifacts. However, in a minority of ICA components with a clearly recognizable cardio-ballistic pattern, a high degree of spatial correlation between along-bundle and across-bundle bipolar measurements was found (Fig. 8a). Since the spatial distribution of these components was insensitive to the cable arrangement they probably did not result from head or conductors/leads motion. Thus, it is likely that the sources of these components were located inside the head. One of the possible explanations of this observation is the Hall Effect (Mullinger et al., 2013). The "inside head" BCG components were marked as a "brain signal" by spatial ICA component classification (criteria 1 and 2). Therefore, we recommend that in addition to the spatial classification, the ICA components should be classified according to their temporal correlation to ECG (as was performed in this study by adding criterion 3 ). Thus, BCG interference is suppressed here using both spatial and temporal classification, whereas non-BCG interference was suppressed using only spatial classification.

While the current work concentrated on the EEG quality, we also evaluated the corresponding fMRI maps. Of the ten patients evaluated, four had subsequently underwent invasive procedures (either invasive electrophysiological monitoring or seizure focus resection) allowing validation of the fMRI results. fMRI maps created with the use of daEEG were concordant with the invasive validation in three of the four patients.

Despite its advantages, several limitations of daEEG should be mentioned. One disadvantage of the daEEG setup used in this research is its application of non-standard EEG electrode locations. In order to obtain equal electrode pair distances between along- and across-measurements, in the daEEG cap used in the present work, half of the electrodes were shifted $2-3 \mathrm{~cm}$ foreword and to the left resulting in non-standard positions. However, with some modifications that do not change the principles of daEEG, an EEG cap can be constructed with standard electrode positions (see Supplementary materials Fig. S6 and Appendix 2). Still, the bundling of cables in the commercially available EEG arrays must be changed for daEEG use. Second, due to the demand for both along and across measurements, daEEG requires a relatively high density electrode array (in our set-up - 64 electrodes). Third, while 64 channels have sufficient electrode density to delineate the topography of most epileptic spikes, it is possible that in some rare cases very focal spikes appear differently on adjacent rows of electrodes. In such a case the correlation criterion for independent component selection would be insufficient. The knowledge of out-of-scanner topography of such spikes can help to distinguish them from artifacts. Fourth, in the current approach, the optimal thresholds for differentiating signal from noise were manually selected. It is clear that subjectivity is the main problem of such a threshold selection approach. In the future, an automatic algorithm for selecting optimal thresholds should be developed. Finally, since the daEEG algorithm employs ICA decomposition, it is sensitive to its drawbacks. ICA also has the potential to mix separate sources into united components, when its basic assumptions are not completely met (James and Hesse, 2005). In the present study we did not show the contribution of daEEG to solving this problem (details are presented in Supplementary material, Appendix 1), but further work is needed to fully evaluate this possibility or develop other solutions for it.

One should mention several limitations of the techniques used in this study to evaluate the efficacy of daEEG and OBS, with particular emphasis on the hybrid analysis. It should be taken into account that the hybrid trace is an artificial model. Inserting one-second data segments from one recording to a different one, may result in the allocation of these epochs to a separate ICA component. This could make it easy for the algorithm to recognize these implanted periods without the need for daEEG technology. However, inspecting the independent components after ICA of the hybrid traces, we observed that the inserted spikes and the natural spikes were generally allocated to the same components (an example is provided in the Supplementary Fig. S2a-d). Additionally, the peak-to-peak amplitudes of the hybrid analysis were slightly inflated following both cleaning procedures evaluated but more so following OBS. As several studies have noted, OBS can in fact add noise to the data instead of removing it. For example, LeVan et al. (2013) found distortions of the EEG data following OBS in the form of attenuation of alpha oscillations and Liu et al. (2012) found that OBS induced spurious lowfrequency (3-8 Hz) components that were absent in the clean EEG spectrum. These distortions may be due to the suboptimal choice of the number of components used (LeVan et al., 2013) or suboptimal detection of QRS events (Vanderperren et al., 2010). Alternatively, this increase in peak-to-peak amplitude can also be a result of the hybrid procedure itself. Since the hybrid epochs are a summation of two signals, this process may raise the overall noise (or background activity) and this in turn has the potential to increase the peak-to-peak measures. Thus, with regards to the peak-to-peak measures of the hybrid analysis it is not yet known whether daEEG performs better than OBS in removing movement related noise or whether OBS adds noise to the signal. In any case, daEEG restores SNR to higher level than OBS, and since SNR is more related to detectability, we conclude that improved detectability can be achieved using daEEG. A more systematic analysis of hybrid traces as a tool for an assessment of signal/noise separation should be performed in future studies.

The conclusion following this study is that the daEEG-fMRI approach can provide a relatively clean EEG signal recorded simultaneously with fMRI. We demonstrated the utility of this method in ten patients with epilepsy, however daEEG can be used more broadly for analyzing different types of brain electrical signals. daEEG can be particularly advantageous in analyzing spontaneous brain activity, in which the SNR cannot be improved by data averaging. We believe that sleep research and the study of resting state brain activity, among others, could be good candidates for daEEG application.

Supplementary data to this article can be found online at http://dx. doi.org/10.1016/j.neuroimage.2016.07.014.

\section{Disclosure}

Ilana Klovatch-Podlipsky, Firas Fahoum, Talma Hendler and Mordekhay Medvedovsky are authors of patent: Device for use in electro-biological signal measurement in the presence of a magnetic field, WO2012046237, 12.04.2012. Other authors report no conflict of interest.

\section{Funding}

This research has been supported by the European Union ACTIVE grant (FP7-ICT-2009-6-270460), the Israel Centers of Research 
Excellence for cognitive studies (no. 51/11, to TH), the Israeli Ministry of Science, Technology and Space (Grant no. 3-11170, to TH.) and the European Union Seventh Framework Programme (FP7/2007-2013) under grant agreement no. 604102 (Human Brain Project).

\section{Acknowledgements}

The authors would like to thank Prof. Jean Gotman and Prof. Amos Korczyn for revising this manuscript, Dr. Dana Ekstein and Dr. Ilan Blatt for referring the patients for our study, Dr. Dafna Ben Bashat and Dr. Moran Artzi for supporting our work in technical aspects of MRI scanning and Mrs. Vicky Myers, Mrs. Yael Yaacob and Mrs. Aliya Solski for their help in editing the manuscript.

\section{References}

Allen, P.J., Polizzi, G., Krakow, K., Fish, D.R., Lemieux, L., 1998. Identification of EEG events in the MR scanner: the problem of pulse artifact and a method for its subtraction. NeuroImage 8, 229-239.

Allen, P.J., Josephs, O., Turner, R., 2000. A method for removing imaging artifact from continuous EEG recorded during functional MRI. NeuroImage 12, 230-239.

Bell, A.J., Sejnowski, T.J., 1995. An information maximization approach to blind separation and blind deconvolution. Neural Comput. 7, 1129-1159.

Bonmassar, G., Purdon, P.L., Jaaskelainen, I.P., Chiappa, K., Solo, V., Brown, E.N., Belliveau, J.W., 2002. Motion and ballistocardiogram artifact removal for interleaved recording of EEG and EPs during MRI. Neurolmage 16, 1127-1141.

Chowdhury, M.E., Mullinger, K.J., Glover, P., Bowtell, R., 2014. Reference layer artefact subtraction (RLAS): a novel method of minimizing EEG artefacts during simultaneous fMRI. Neurolmage 84, 307-319.

Debener, S., Mullinger, K.J., Mazy, R.K., Bowtell, R.W., 2008. Properties of the ballistocardiogram artefact as revealed by EEG recordings at $1.5,3$ and $7 \mathrm{~T}$ static magnetic field strength. Int. J. Psychophysiol. 67, 189-199.

Delorme, A., Makeig, S., 2004. EEGLAB: an open source toolbox for analysis of single-trial EEG dynamics including independent component analysis. J. Neurosci. Methods 134, 9-21.
Huiskamp, G., 2006. Reduction of the ballistocardiogram artifact in simultaneous EEGfMRI using ICA. Engineering in Medicine and Biology Society, 2005. IEEE-EMBS 2005. 27th Annual International Conference of the IEEE, pp. 3691-3694.

James, C.J., Hesse, C.W., 2005. Independent component analysis for biomedical signals. Physiol. Meas. 26, R15-R39.

Jorge, J., Grouiller, F., Gruetter, R., van der Zwaag, W., Figueiredo, P., 2015. Towards highquality simultaneous EEG-fMRI at $7 \mathrm{~T}$ : detection and reduction of EEG artifacts due to head motion. NeuroImage 120, 143-153.

Kobayashi, E., Bagshaw, A.P., Grova, C., Dubeau, F., Gotman, J., 2006. Negative BOLD responses to epileptic spikes. Hum. Brain Mapp. 27, 488-497.

LeVan, P., Maclaren, J., Herbst, M., Sostheim, R., Zaitsev, M., Hennig, J., 2013. Ballistocardiographic artifact removal from simultaneous EEG-fMRI using an optical motion-tracking system. NeuroImage 75, 1-11.

Liu, Z.M., de Zwart, J.A., van Gelderen, P., Kuo, L.W., Duyn, J.H., 2012. Statistical feature extraction for artifact removal from concurrent fMRI-EEG recordings. Neurolmage 59, 2073-2087.

Luo, Q., Huang, X., Glover, G.H., 2014. Ballistocardiogram artifact removal with a reference layer and standard EEG cap. J. Neurosci. Methods 233, 137-149.

Mantini, D., Perrucci, M.G., Cugini, S., Ferretti, A., Romani, G.L., Del Gratta, C., 2007. Complete artifact removal for EEG recorded during continuous fMRI using independent component analysis. NeuroImage 34, 598-607.

Masterton, R.A Abbott, D.F. Fleming. S.W., Jackson, G.D., 2007. Measurement and reduction of motion and ballistocardiogram artefacts from simultaneous EEG and fMRI recordings. NeuroImage 37, 202-211.

Mullinger, K.J., Havenhand, J., Bowtell, R., 2013. Identifying the sources of the pulse artefact in EEG recordings made inside an MR scanner. NeuroImage 71, 75-83.

Niazy, R.K., Beckmann, C.F., Iannetti, G.D., Brady, J.M., Smith, S.M., 2005. Removal of FMRI environment artifacts from EEG data using optimal basis sets. NeuroImage 28 720-737.

Srivastava, G., Crottaz-Herbette, S., Lau, K.M., Glover, G.H., Menon, V., 2005. ICA-based procedures for removing ballistocardiogram artifacts from EEG data acquired in the MRI scanner. NeuroImage 24, 50-60.

Vanderperren, K., De Vos, M., Ramautar, J.R., Novitskiy, N., Mennes, M., Assecondi, S., Vanrumste, B., Stiers, P., Van den Bergh, B.R.H., Wagemans, J., Lagae, L., Sunaert, S., Van Huffel, S., 2010. Removal of BCG artifacts from EEG recordings inside the MR scanner: a comparison of methodological and validation-related aspects. NeuroImage 50, 920-934. 